\title{
Serum and Tissue Levels of Advanced Glycation End Products and Risk of Mortality in Patients on Maintenance Hemodialysis
}

\author{
Jianping Jiang ${ }^{a}$ Yuanyuan Zhang $^{\text {a }}$ Jianghua Chen ${ }^{b} \quad$ Xiaobing Yang $^{a}$ \\ Changlin Mei $^{c}$ Fei Xiong ${ }^{d}$ Wei Shi ${ }^{\text {e }}$ Wei Zhou $^{f}$ Xusheng Liu ${ }^{g}$ Shiren Sun ${ }^{\mathrm{h}}$ \\ Ping Zhang ${ }^{\mathrm{b}}$ Yixiang Zhang $^{\mathrm{c}}$ Yanmin Zhang $^{\mathrm{d}}$ Shuangxin Liu ${ }^{\mathrm{e}}$ \\ Zhimin Zhang $^{f}$ Qizhan Ling ${ }^{\mathrm{Y}}$ Yan Yu $^{\mathrm{h}}$ Jianwei Tian ${ }^{\mathrm{a}}$ Weihong Luo ${ }^{\mathrm{a}}$ \\ Xianhui Qina Fan Fan Hou ${ }^{a}$ \\ aDivision of Nephrology, Nanfang Hospital, Southern Medical University, National Clinical Research Center for \\ Kidney Disease, State Key Laboratory of Organ Failure Research, Guangdong Provincial Institute of Nephrology, \\ Guangdong Provincial Key Laboratory of Renal Failure Research, Guangzhou Regenerative Medicine and Health

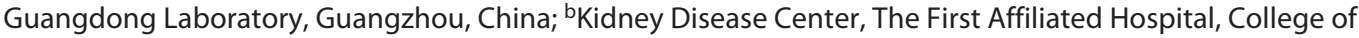 \\ Medicine, Zhejiang University, Hangzhou, China; 'Division of Nephrology, Changzheng Hospital, Shanghai, China; \\ ${ }^{d}$ Department of Nephrology, Wuhan No. 1 Hospital, Tongji Medical College, Huazhong University of Science and \\ Technology, Wuhan, China; 'Department of Nephrology, Guangdong Provincial People's Hospital, Guangdong \\ Academy of Medical Sciences, Guangzhou, China; fDepartment of Nephrology, The 8th Medical Center of Chinese \\ PLA General Hospital, Beijing, China; ${ }^{9}$ Department of Nephrology, Guangdong Provincial Hospital of Traditional \\ Chinese Medicine, Second Clinical Medical College, Guangzhou University of Chinese Medicine, Guangzhou, China;

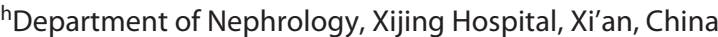

\section{Keywords}

Skin autofluorescence $\cdot \mathrm{N}^{\varepsilon}$-carboxymethyl-lysine $\cdot$ All-cause mortality $\cdot$ Cardiovascular diseases mortality $\cdot$ Hemodialysis patients

\begin{abstract}
Background: The relation of tissue and circulating advanced glycation end products (AGEs) with mortality in hemodialysis (HD) patients remains inconclusive. We aimed to investigate the association of serum AGEs (CML) and tissue AGEs estimated by skin autofluorescence (SAF) with all-cause and cardiovascular disease (CVD) mortality, and examine the possible modifiers for the association in HD patients with by far the largest sample size in any similar studies. Methods: A
\end{abstract}

total of 1,634 HD patients were included from the China Cooperative Study on Dialysis (CCSD), a multicenter prospective cohort study. The primary and secondary outcomes were all-cause mortality and CVD mortality, respectively. $\boldsymbol{R} \boldsymbol{e}$ sults: The median follow-up duration was 5.2 years. Overall, there was a positive relation of baseline SAF levels with the risk of all-cause mortality (per $1 \mathrm{AU}$ increment, adjusted hazard ratio (HR), 1.30; 95\% confidence interval $(\mathrm{Cl}): 1.12,1.50)$ and CVD mortality (per 1 AU increment, adjusted HR, 1.36; $95 \% \mathrm{Cl}: 1.14,1.62$ ). Moreover, a stronger positive association between baseline SAF (per $1 \mathrm{AU}$ increment) and all-cause mortality was found in participants with shorter dialysis vin-

Jiang J. and Zhang Y. contributed equally to this work. Hou F.F. and Qin X. contributed equally to this work. karger@karger.com

www.karger.com/ajn

Karger $\stackrel{2}{=}$
(C) 2021 S. Karger AG, Basel
Fan Fan Hou

Division of Nephrology, Nanfang Hospital Southern Medical University 1838 North Guangzhou Ave.

Guangzhou 510515 (China)

ffhouguangzhou@163.com 
tage, or lower C-reactive protein levels (Both $p$ interactions $<0.05)$. Nevertheless, there was no significant association between serum CML and the risk of mortality. Conclusions: In patients undergoing long-term $\mathrm{HD}$, baseline $\mathrm{SAF}$, but not serum $C M L$, was significantly associated with the risk of allcause and CVD death.

(c) 2021 S. Karger AG, Basel

\section{Introduction}

Hemodialysis (HD) is a life-sustaining treatment for patients with ESRD. HD patients have a significantly increased risk of mortality than the general population $[1,2]$. The leading cause of mortality in HD patients is cardiovascular disease (CVD), which is associated with not only the traditional risk factors but also a number of nontraditional risk factors, such as anemia, parathyroid hormone, mineral bone disorders, and uremic toxins [3-5].

Advanced glycation end products (AGEs) have been identified as uremic toxins, formed by the nonenzymatic reaction (the Maillard reaction) between sugars and the free amino groups of proteins, lipids, or nucleic acids [6]. HD patients show markedly increased AGE levels due to decreased renal clearance and increased endogenous formation [7]. Accumulation of AGEs has been indicated as a mechanism underlying inflammation, oxidative stress, and structural tissue damage leading to vascular diseases or mortality [8].

However, results from previous prospective studies of circulating AGE levels in HD patients have been conflicting, ranging from a protective factor [9], no clear association [10], or a risk factor of mortality [11]. Most of these studies did not measure the tissue level of AGEs, a better mirror of AGE-dependent tissue damage [12]. The development of skin autofluorescence (SAF) provided a noninvasive, easy-to-operate, and inexpensive method for measurement of tissue AGE levels [13]. However, few studies have observed the level of serum and tissue AGEs simultaneously and separately analyzed their impacts on the risk of mortality in patients undergoing long-term dialysis. The present study, using data from a multicenter cohort of 1,634 HD patients with a median follow-up of 5.2 years, investigates the association of serum and tissue AGEs with all-cause and CVD mortality, and examines the possible modifiers for the association.

Tissue AGEs and Mortality in HD Patients

\section{Materials and Methods}

\section{Study Population and Design}

The current cohort study consisted of patients enrolled in the China Cooperative Study on Dialysis (CCSD). The baseline data of the CCSD had been reported previously [14-17]. In brief, the CCSD is performed in 9 of the largest dialysis centers (at least $200 \mathrm{HD}$ patients in each center) in 6 cities of China (Beijing, Shanghai, Guangzhou, Hangzhou, Wuhan, and Xi'an). Eligible participants were men and women aged $\geq 18$ years with ESRD undergoing dialysis between January 1, 2005 and December 1, 2010. A total of $2,183 \mathrm{HD}$ patients were screened in the CCSD [16]. The current cohort study enrolled 1,634 eligible HD participants from the CCSD, followed up from July 2010 to February 2016.

\section{Data Collection, Measurements, and Follow-Up}

Baseline data used in the present study were derived from the database of the CCSD. All data were collected at enrollment on the bases of review of medical records by a group of experienced doctors and research nurses. The following data were collected: demographic data, underlying renal diseases, medication records, dialysis modality, dialysis program, and CVD, which was defined as the presence of clinically diagnosed ischemic heart disease, heart failure, and/or stroke after initiation of dialysis.

Blood pressure (BP) measurement was taken by using a sphygmomanometer before each of the $3 \mathrm{HD}$ sessions, 3 times at 1-min intervals, all after $10 \mathrm{~min}$ of rest in a supine decubitus position. The mean of the 3 readings was calculated.

Participants were scheduled for a follow-up every 1-3 months in each center. At each follow-up visit, possible end point events were documented by trained research staff and physicians.

\section{Hemodialysis Regimens}

HD patients were dialyzed twice or thrice weekly with low-flux polysulphone or polyacrylamide dialyzer, either 1.5 or $1.7 \mathrm{~m}^{2}$ (Fresenius, Germany; Gambro, Sweden; Nipro, Japan; B. Braun, Germany; Langsheng, China). All treatments were of 4- to 5-h duration with conventional glucose-free, bicarbonate-based dialysate containing 1.25-1.5 mM calcium, $2.0 \mathrm{~mm}$ potassium, and $138 \mathrm{mM}$ sodium. Dialysate flow was $500 \mathrm{~mL} / \mathrm{min}$.

\section{Laboratory Assays}

Baseline fasting venous serum samples were collected prior to the HD sessions. Biochemical tests were performed using automatic clinical analyzers following the same standard protocol at each local dialysis center.

Determination of serum CML (a component of AGEs) was based on spectrofluorometric detection [18] at the core laboratory of Nanfang Hospital, Guangzhou, China. SAF was measured before the latest HD session follow-up by using a cutaneous autofluorescence device (AGE Reader; DiagnOptics Technologies, The Netherlands) [13]. The schematic diagram of SAF measurements is shown in online suppl. Fig. 1 (For all online suppl. material, see www.karger.com/doi/10.1159/000512385.) The values were compared with an age-matched non-CKD database contained within the device. The autofluorescence reader illuminates a skin surface of $\sim 1 \mathrm{~cm}^{2}$, guarded against surrounding light, with an excitation light source between 300 and $420 \mathrm{~nm}$ (peak excitation approximately $350 \mathrm{~nm}$ ). Only light from the skin is measured with a spec- 
trometer in the 300 - to $600-\mathrm{nm}$ range, using a $200-\mathrm{mm}$ glass fiber (Farnell, Leeds, UK). All measurements were performed at room temperature in a semi-dark environment before dialysis. The nondominant forearm rests on the device, and 3 readings, all taken within $1 \mathrm{~cm}$ of each other away from any areas of bruising or pigmentation, were averaged and recorded. A connected computer analyzed the level of autofluorescence and correlated that to know the normal range. Repeated autofluorescence measurements on 1 day and intraindividual seasonal variance showed an Altman error percentage of $<6$. The intra- and inter-day assay precision expressed as coefficients of variation for autofluorescence measurements were 2.5 and $4.6 \%$, respectively.

\section{Outcomes}

All-cause mortality was the primary outcome, which included death due to any reason. The secondary outcome was CVD mortality, which included sudden cardiac death, stroke, myocardial infarction, heart failure, and death due to other known vascular causes. Evidence for death included death certificates from hospitals or reports from investigator visits.

\section{Statistical Analysis}

We assumed that the annual mortality rate of HD patients with low SAF levels was about $3 \%$, with an alpha of 0.05 , enrolled about 1,600 HD patients (high vs. low SAF: about 3:1) followed up for 5 years, would provide us $>80 \%$ power to detect an effect of a hazard ratio (HR) of $>1.5$ between high and low SAF groups.

Baseline characteristics are presented as means \pm standard deviations (SDs) or medians (interquartile range) and proportions for continuous and categorical variables, respectively. Statistical significance of differences in baseline characteristics was assessed in accordance with baseline SAF quartiles $(<2.4,2.4$ to $<2.9,2.9$ to $<3.5, \geq 3.5 \mathrm{AU}$ ) using ANOVA tests or $\chi^{2}$ tests, accordingly.

The relation of baseline SAF levels with all-cause and CVD mortality was explored using thin plate regression splines in generalized additive models implemented by the R package $m g c v$. To evaluate and compare the impact of baseline SAF and CML on study outcomes, SAF and CML were first individually and then simultaneously entered into the Cox proportional hazard regression models without and with adjustment for study centers, age, sex, smoking status, BMI, systolic BP, dialysis vintage, albumin, hemoglobin, intact parathyroid hormone, phosphate, calcium, sodium, potassium, fasting glucose, total cholesterol, CVD status, and the usage of renin-angiotensin-aldosterone system inhibitors at baseline. Our analysis showed that there was no obvious multicollinearity among the variables (online suppl. Table 1). The Kaplan-Meier curves were used to visualize and compare the cumulative hazards for all-cause and CVD mortality by baseline SAF categories. In addition, possible modifications to the association of baseline SAF levels with all-cause mortality were evaluated by stratified analyses and interaction testing. We also tested whether SAF improved 3- and 4-year risk prediction by comparing the performance of risk prediction models with versus without SAF using R package survcomp.

A 2-tailed $p<0.05$ was considered statistically significant in all analyses. R software, version 3.6.3 (http://www.R-project.org/), was used to perform all statistical analyses.

\section{Results}

\section{Characteristics of Study Participants}

As illustrated in the flowchart of the study participants (online suppl. Fig. 2), of the 1,835 participants in the follow-up study, a total of 1,634 HD patients without a switch to PD, kidney transplantation, or lost to follow-up during the follow-up period were included in the final analysis.

The mean SAF and serum CML levels of the patients were $3.0 \pm 0.8 \mathrm{AU}$ and $154.3 \pm 43.0 \mu \mathrm{mol} / \mathrm{L}$, respectively. The mean age was $57.4 \pm 14.6$ years, with a mean dialysis vintage of $50.0 \pm 48.4$ months. Baseline characteristics of the patients by SAF quartiles $(<2.4,2.4$ to $<2.9,2.9$ to $<3.5$, $\geq 3.5 \mathrm{AU})$ and CML quartiles $(<123.7,123.7$ to $<151.1$, 151.1 to $<181.9, \geq 181.9 \mu \mathrm{mol} / \mathrm{L}$ ) are presented in Table 1 and online suppl. Table 2, respectively. The univariate analysis showed that there were significant differences in a lot of variables by SAF quartiles. The multivariable analysis further found that there was a positive relation of SAF with age, dialysis vintage, and CVD status; and a negative association of SAF with DBP and HDL cholesterol (online suppl. Table 3).

\section{Association between Baseline SAF and Study \\ Outcomes}

During a median follow-up of 5.2 years (interquartile range, 3.2-5.3), all-cause or CVD death occurred in 553 (33.8\%) and 375 (22.9\%) patients, respectively. Overall, there was a positive relationship of baseline SAF levels with the risk of all-cause mortality (per $1 \mathrm{AU}$ increment, adjusted oddsHR, 1.30; 95\% confidence interval [CI]: 1.12, 1.50) (Fig. 1a). When baseline SAF levels were assessed as quartiles, the adjusted HRs and $95 \% \mathrm{CI}$ in the second, third, and fourth quartiles were $2.06(1.35,3.14)$, $2.14(1.41,3.24)$, and $2.49(1.64,3.79)$, respectively, when compared with quartile 1 ( $p$ for trend $<0.001$ ). KaplanMeier curves of the cumulative event rate of all-cause mortality for the SAF categories ( $<2.4$ vs. $\geq 2.4$ AU) are shown in online suppl. Figure 3a. Consistently, a significantly higher risk of all-cause mortality (adjusted HR, $2.20 ; 95 \%$ CI: $1.51,3.22$ ) was found in patients in quartiles 2-4 than those in quartile 1 (Table 2).

Similarly, there was a positive association between baseline SAF levels and CVD mortality (per 1 AU increment, adjusted HR, 1.36; 95\% CI: 1.14, 1.62) (Fig. 1b). Accordingly, participants in quartiles 2-4 had a significantly higher risk of CVD mortality (adjusted HR, 2.12; 95\% CI: $1.35,3.35$ ) than with those in quartile 1 (Table 2 ; online suppl. Fig. 3b). In addition, further adjustment for 
Table 1. Baseline characteristics of study participants by SAF quartiles ${ }^{a}$

\begin{tabular}{|c|c|c|c|c|c|}
\hline \multirow[t]{2}{*}{ Variable } & \multicolumn{4}{|l|}{ SAF, AU } & \multirow[t]{2}{*}{$p$ value } \\
\hline & Q1 $(<2.4)$ & Q2 $(2.4$ to $<2.9)$ & Q3 $(2.9$ to $<3.5)$ & $\mathrm{Q} 4(\geq 3.5)$ & \\
\hline Male, $n(\%)$ & $214(56.5)$ & $221(59.7)$ & $240(54.3)$ & $234(52.8)$ & 0.225 \\
\hline Age, years & $50.4 \pm 14.8$ & $55.4 \pm 14.5$ & $59.2 \pm 13.2$ & $63.4 \pm 13.0$ & $<0.001$ \\
\hline Dialysis vintage, months & $38.8 \pm 40.0$ & $48.7 \pm 47.6$ & $47.6 \pm 42.7$ & $62.9 \pm 57.3$ & $<0.001$ \\
\hline $\mathrm{SBP}, \mathrm{mm} \mathrm{Hg}$ & $143.9 \pm 21.8$ & $143.2 \pm 22.5$ & $142.5 \pm 22.6$ & $139.9 \pm 22.6$ & 0.054 \\
\hline Diastolic BP, mm Hg & $85.3 \pm 13.7$ & $82.9 \pm 13.2$ & $80.9 \pm 11.5$ & $77.4 \pm 11.8$ & $<0.001$ \\
\hline Smoking, $n(\%)$ & $29(7.7)$ & $49(13.2)$ & $58(13.1)$ & $61(13.8)$ & 0.028 \\
\hline CVD, $n(\%)$ & $146(38.5)$ & $149(40.3)$ & $178(40.3)$ & $226(51.0)$ & $<0.001$ \\
\hline Diabetes, $n(\%)$ & $114(30.1)$ & $139(37.6)$ & $189(42.8)$ & $210(47.4)$ & $<0.001$ \\
\hline Hypertension, $n(\%)$ & $337(88.9)$ & $344(93.0)$ & $404(91.4)$ & $400(90.3)$ & 0.257 \\
\hline HDL cholesterol, $\mathrm{mmol} / \mathrm{L}$ & $1.1 \pm 0.4$ & $1.1 \pm 0.4$ & $1.1 \pm 0.4$ & $1.1 \pm 0.4$ & 0.082 \\
\hline $\mathrm{FG}, \mathrm{mmol} / \mathrm{L}$ & $5.0 \pm 1.8$ & $5.6 \pm 2.4$ & $5.8 \pm 2.4$ & $5.8 \pm 2.2$ & $<0.001$ \\
\hline $\mathrm{Cr}, \mu \mathrm{mol} / \mathrm{L}$ & $938.5 \pm 376.6$ & $870.5 \pm 348.4$ & $793.3 \pm 346.5$ & $781.5 \pm 333.6$ & $<0.001$ \\
\hline Uric acid, $\mu \mathrm{mol} / \mathrm{L}$ & $422.0 \pm 151.6$ & $403.8 \pm 152.1$ & $380.3 \pm 153.7$ & $377.0 \pm 153.5$ & $<0.001$ \\
\hline Calcium, mmol/L & $2.2 \pm 0.3$ & $2.2 \pm 0.3$ & $2.3 \pm 0.3$ & $2.3 \pm 0.3$ & $<0.001$ \\
\hline Phosphate, mmol/L & $2.0 \pm 0.7$ & $2.0 \pm 0.7$ & $1.9 \pm 0.6$ & $1.8 \pm 0.7$ & $<0.001$ \\
\hline Sodium, $\mathrm{mmol} / \mathrm{L}$ & $140.8 \pm 3.9$ & $139.8 \pm 3.4$ & $139.5 \pm 3.9$ & $139.2 \pm 4.1$ & $<0.001$ \\
\hline Potassium, mmol/L & $5.0 \pm 0.8$ & $4.9 \pm 0.8$ & $5.0 \pm 0.9$ & $4.9 \pm 0.8$ & 0.230 \\
\hline Albumin, g/L & $39.9 \pm 6.0$ & $39.7 \pm 6.6$ & $39.1 \pm 6.7$ & $38.8 \pm 6.4$ & 0.055 \\
\hline Hemoglobin, g/L & $99.6(21.3)$ & $104.4(20.9)$ & $105.2(19.1)$ & $104.1(19.2)$ & $<0.001$ \\
\hline $\mathrm{CRP}, \mathrm{mg} / \mathrm{L}$ & $3.5(2.0-9.7)$ & $3.9(2.1-12.3)$ & $3.7(2.2-9.7)$ & $5.3(2.9-12.2)$ & 0.009 \\
\hline iPTH, pg/mL & $284.0(136.4-562.7)$ & $255.5(128.2-496.2)$ & $238.6(117.4-491.1)$ & $252.1(127.9-586.4)$ & 0.378 \\
\hline
\end{tabular}

BP, blood pressure; SBP, systolic blood pressure; iPTH, intact parathyroid hormone; HDL, high-density lipoprotein; RAAS, reninangiotensin-aldosterone system; SAF, skin autofluorescence; CVD, cardiovascular disease; FG, fasting glucose; TC, total cholesterol; $\mathrm{CRP}, \mathrm{C}$-reactive protein. ${ }^{\mathrm{a}}$ Continuous variables are presented as mean $\pm \mathrm{SD}$, or median (25th-75th percentile). ${ }^{\mathrm{b}} \mathrm{kt} / \mathrm{v}$ was per session in HD.

baseline serum CML (online suppl. Table 4), or baseline $\mathrm{Cr}$ and the use of glucose-lowering drugs, lipid-lowering drugs, and phosphorus binder (online suppl. Table 5), or uric acid (online suppl. Table 6), or dialysis adequacy (online suppl. Table 7) did not substantially change the results.

\section{Association between Baseline Serum CML and Study Outcomes}

Overall, there was no significant relation of baseline serum CML with all-cause mortality (per 1 SD increment, adjusted HR, 1.03; 95\% CI: $0.88,1.20$ ) and CVD mortality (per 1 SD increment, adjusted HR, 0.91; 95\% CI: 0.74, 1.10) (online suppl. Table 8). 


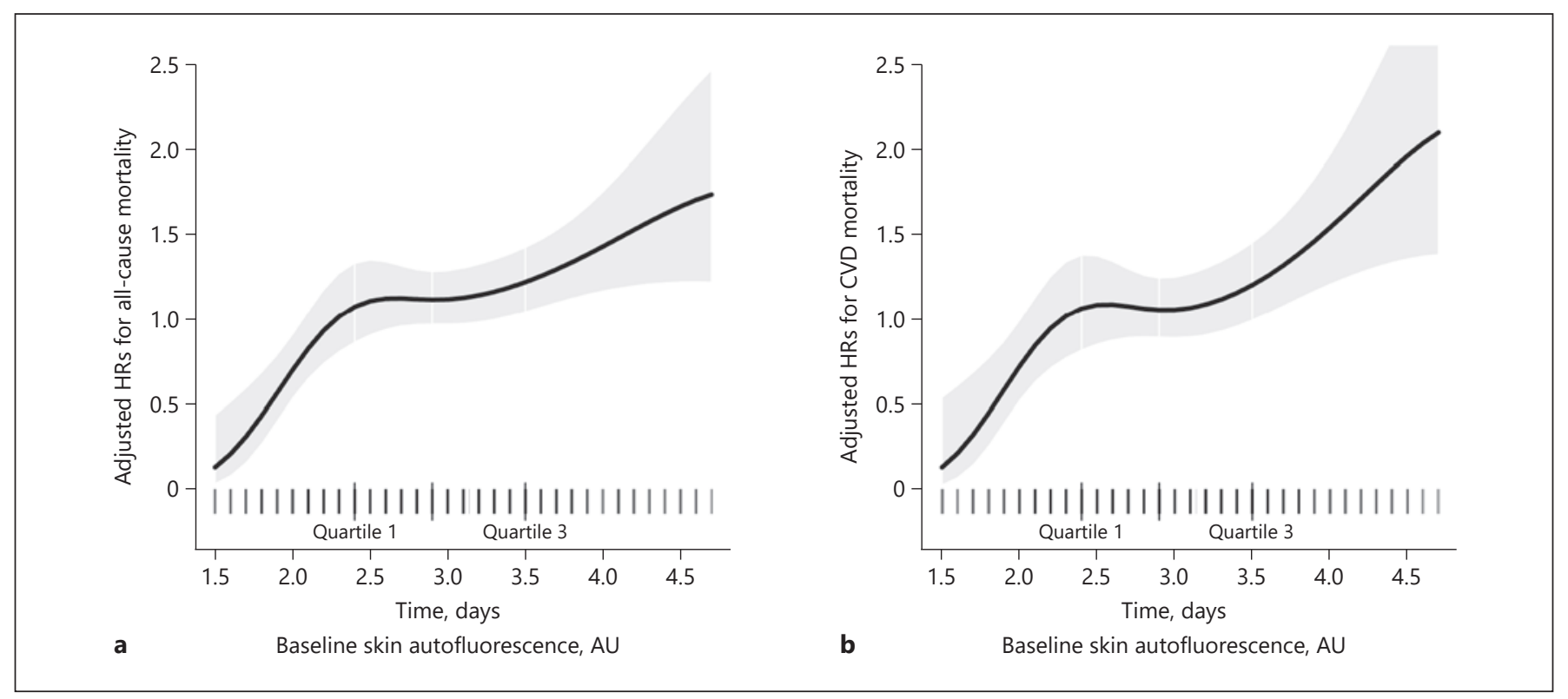

Fig. 1. Relation of baseline SAF with the risk of all-cause mortality (a) and CVD mortality (b) in HD patients. Adjusted for study center, age, sex, smoking status, BMI, SBP, dialysis vintage, albumin, hemoglobin, iPTH, phosphate, calcium, sodium, potassium, FG, TC, CVD status, and the usage of RAAS inhibitors. HR, hazard

Stratified Analyses for the Association of Baseline SAF with All-Cause Mortality

We further performed stratified analyses to assess the relation of baseline SAF (per 1 AU increment) with allcause mortality in various subgroups. A stronger positive association between baseline SAF and all-cause mortality was found in participants with dialysis vintage $<34.0$ months (median) (adjusted HR, 1.51; 95\% CI: 1.25, 1.82 vs. $\geq 34.0$ months: adjusted HR, 1.13; 95\% CI: 0.92, 1.38; $p$ interaction $=0.028)$, or with a C-reactive protein $(\mathrm{CRP})$ level $<11.1 \mathrm{mg} / \mathrm{L}$ (quartile 3) (adjusted HR, 1.44; 95\% CI: $1.17,1.76$ vs. $\geq 11.1 \mathrm{mg} / \mathrm{L}$ : adjusted HR, $1.04 ; 95 \%$ CI: 0.80 , 1.35; $p$ interaction $=0.042)$ (Fig. 2). However, other variables at baseline, including sex, age, dialysis adequacy, systolic BP, diabetes, total cholesterol, albumin, and concomitant CVD, did not significantly modify the association between SAF and the risk of all-cause mortality (all $p$ interactions $>0.05$ ) (Fig. 2).

\section{Assessment of Discrimination and Model Accuracy}

We next compared whether the addition of SAF improved model prediction for the all-cause and CVD mortality. The addition of SAF to the fully adjusted clinical model significantly improved the C-statistic for the 4-year all-cause mortality from 0.72 (95\% CI: $0.69,0.74)$ to 0.73 ratio; $\mathrm{HD}$, hemodialysis; $\mathrm{SAF}$, skin autofluorescence; $\mathrm{CVD}$, cardiovascular disease; SBP, systolic blood pressure; iPTH, intact parathyroid hormone; FG, fasting glucose; TC, total cholesterol; RAAS, renin-angiotensin-aldosterone system.

(95\% CI: 0.70, 0.76, $p=0.009$ ) and improved the prediction of CVD mortality with C-statistics from 0.73 (95\% CI: $0.70,0.76$ ) to 0.74 (95\% CI: $0.70,0.77, p=0.044$ ). Similar results were found for the 3-year all-cause and CVD mortality (online suppl. Table 9). However, the addition of SAF to the clinical model only resulted in a slightly greater improvement in risk reclassification (online suppl. Table 10).

\section{Discussion}

Our study demonstrated that there was a positive relation of baseline tissue AGE levels with all-cause and CVD mortality, independent of circulating AGEs and other important confounders, among patients undergoing long-term HD. Moreover, our study expanded the results of previous studies by demonstrating that the positive relation between tissue AGEs and all-cause mortality was more pronounced in patients with shorter dialysis vintage or lower CRP levels, suggesting that the accumulative level of tissue AGEs might be an early predictor for the risk of all-cause or CV death.

Previous studies have linked the tissue AGEs with allcause and/or CVD mortality in HD patients, but the re-
12

Am J Nephrol 2021;52:8-16

DOI: $10.1159 / 000512385$
Jiang et al. 
Table 2. Association between SAF and study outcomes

\begin{tabular}{|c|c|c|c|c|c|c|}
\hline \multirow[t]{2}{*}{ Skin AGE, AU } & \multirow[t]{2}{*}{$N$} & \multirow{2}{*}{$\begin{array}{l}\text { Events } \\
(\%)\end{array}$} & \multicolumn{2}{|l|}{ Crude model } & \multicolumn{2}{|l|}{ Adjusted model $^{\mathrm{a}}$} \\
\hline & & & HR (95\% CI) & $p$ value & HR (95\% CI) & $p$ value \\
\hline \multicolumn{7}{|l|}{ All-cause mortality } \\
\hline Continuous & 1,634 & $553(33.8)$ & $1.51(1.38,1.67)$ & $<0.001$ & $1.30(1.12,1.50)$ & $<0.001$ \\
\hline \multicolumn{7}{|l|}{ Quartiles } \\
\hline $\mathrm{Q} 1(<2.4)$ & 379 & $67(17.7)$ & 1.00 (ref.) & & 1.00 (ref.) & \\
\hline Q2 $(2.4$ to $<2.9)$ & 370 & $122(33.0)$ & $2.01(1.50,2.71)$ & $<0.001$ & $2.06(1.35,3.14)$ & $<0.001$ \\
\hline Q3 $(2.9$ to $<3.5)$ & 442 & $163(36.9)$ & $2.29(1.72,3.04)$ & $<0.001$ & $2.14(1.41,3.24)$ & $<0.001$ \\
\hline $\mathrm{Q} 4(\geq 3.5)$ & 443 & $201(45.4)$ & $3.02(2.29,3.99)$ & $<0.001$ & $2.49(1.64,3.79)$ & $<0.001$ \\
\hline$p$ for trend & & & $<0.001$ & & $<0.001$ & \\
\hline \multicolumn{7}{|l|}{ Categories } \\
\hline Q1 $(<2.4)$ & 379 & $67(17.7)$ & 1.00 (ref.) & & 1.00 (ref.) & \\
\hline Q2-4 ( $\geq 2.4)$ & 1,255 & $486(38.7)$ & $2.45(1.90,3.16)$ & $<0.001$ & $2.20(1.51,3.22)$ & $<0.001$ \\
\hline \multicolumn{7}{|l|}{ CVD mortality } \\
\hline Continuous & 1,634 & $375(22.9)$ & $1.50(1.34,1.69)$ & $<0.001$ & $1.36(1.14,1.62)$ & $<0.001$ \\
\hline \multicolumn{7}{|l|}{ Quartiles } \\
\hline $\mathrm{Q} 1(<2.4)$ & 379 & $46(12.1)$ & 1.00 (ref.) & & 1.00 (ref.) & \\
\hline Q2 $(2.4$ to $<2.9)$ & 370 & $84(22.7)$ & $2.02(1.41,2.90)$ & $<0.001$ & $1.88(1.13,3.14)$ & 0.015 \\
\hline Q3 $(2.9$ to $<3.5)$ & 442 & $112(25.3)$ & $2.29(1.62,3.23)$ & $<0.001$ & $2.17(1.32,3.58)$ & 0.002 \\
\hline $\mathrm{Q} 4(\geq 3.5)$ & 443 & $133(30.0)$ & $2.92(2.08,4.08)$ & $<0.001$ & $2.43(1.46,4.04)$ & $<0.001$ \\
\hline$p$ for trend & & & $<0.001$ & & 0.001 & \\
\hline \multicolumn{7}{|l|}{ Categories } \\
\hline Q1 (<2.4) & 379 & $46(12.1)$ & 1.00 (ref.) & & 1.00 (ref.) & \\
\hline Q2-4 ( $\geq 2.4)$ & 1,255 & $329(26.2)$ & $2.42(1.77,3.29)$ & $<0.001$ & $2.12(1.35,3.35)$ & 0.001 \\
\hline
\end{tabular}

SAF, skin autofluorescence; iPTH, intact parathyroid hormone; SBP, systolic blood pressure; FG, fasting glucose; CVD, cardiovascular disease; TC, total cholesterol; RAAS, renin-angiotensin-aldosterone system; HR, hazard ratio; CI, confidence interval; AGE, advanced glycation end product. ${ }^{\text {a }}$ Adjusted for study centers, age, sex, smoking status, SBP, dialysis vintage, albumin, hemoglobin, iPTH, phosphate, calcium, sodium, potassium, FG, TC, CVD status, and the usage of RAAS inhibitors.

ported results are inconsistent [19-24]. Some studies suggest a positive association between SAF levels and allcause [19-23] or CVD mortality [19, 20, 24], while others find no significant impacts of tissue AGEs on CVD mortality $[21,22]$. A recent study [25] in patients with CKD stage 5 indicates that the skin AGE level is not associated with all-cause mortality or CVD mortality in the fully adjusted models. Of note, all the previous studies had a smaller sample size $(n=105-332)$ [19-25] and could be defined as exploratory. To overcome the problem, a meta-analysis [26] was conducted and showed that higher SAF levels were associated with the risk of CVD $(R R, 1.97$; 95\% CI: 1.11-3.49) and all-cause mortality (RR, 2.08; 95\% CI: 1.41-3.06) in HD patients. However, the obvious heterogeneity across the studies (I-square $=62.4 \% ; p=0.046$ ) in the meta-analysis suggests that the results may be dominated by the studies with relatively larger sample size and are unreliable. These results call for a large-scale cohort study to further confirm the association between tissue AGEs and the risk of mortality in HD patients.

Our study provided a chance to evaluate the dose-response relation of SAF levels with mortality in HD patients with by far the largest sample size $(n=1,634)$ in many similar studies and included a comprehensive adjustment and stratified analysis for the important confounders. In the current study, the annual mortality rate in low-SAF patients was $3.4 \%$, and a sample size of 1,634 (high vs. low SAF levels: 3:1), with a type I error rate of $5 \%$, provide us $>90 \%$ power to detect an effect of an HR of 2.2 (high vs. low SAF levels) during the 5.2-year followup.

Our study provided some new insights. First, we demonstrated that higher SAF associated with increased allcause and CVD mortality in HD patients, independent of serum CML and other traditional or suspected risk factors. Consistently, previous studies have reported that tis- 


\begin{tabular}{|c|c|c|c|c|c|}
\hline \multicolumn{5}{|c|}{ Skin autofluorescence, AU Size No. of events (\%) adjusted OR $(95 \% \mathrm{CI})^{\mathrm{a}}$} & \multirow{2}{*}{$\begin{array}{l}\text { P-interaction } \\
\\
0.548\end{array}$} \\
\hline Age, years & & & & & \\
\hline$<60$ & 906 & 187 (20.6) & $1.43(1.09,1.88)$ & $\longrightarrow$ & \\
\hline$\geq 60$ & 728 & $366(50.3)$ & $1.30(1.11,1.53)$ & - & \\
\hline Sex & & & & & 0.983 \\
\hline Male & 909 & $327(36.0)$ & $1.30(1.09,1.54)$ & $\rightarrow$ & \\
\hline Female & 725 & $226(31.2)$ & $1.30(1.04,1.63)$ & $\rightarrow$ & \\
\hline Dialysis vintage, mon & & & & & 0.028 \\
\hline$<34.0$ (median) & 817 & $258(31.6)$ & $1.51(1.25,1.82)$ & $\rightarrow$ & \\
\hline$\geq 34.0$ & 817 & $295(36.1)$ & $1.13(0.92,1.38)$ & $=$ & \\
\hline Dialysis adequacy, kt/v & & & & & 0.416 \\
\hline$<1.5$ (median) & 388 & 154 (39.7) & $1.23(0.96,1.58)$ & $=$ & \\
\hline$\geq 1.5$ & 400 & $135(33.8)$ & $1.06(0.80,1.41)$ & {[} & \\
\hline $\mathrm{SBP}, \mathrm{mm} \mathrm{Hg}$ & & & & & 0.248 \\
\hline$<140$ & 671 & $227(33.8)$ & $1.19(0.98,1.46)$ & $=$ & \\
\hline$\geq 140$ & 963 & 326 (33.9) & $1.39(1.15,1.68)$ & $\rightarrow$ & \\
\hline Total cholesterol, mmol/L & & & & & 0.615 \\
\hline$<4.0$ (median) & 672 & $222(33.0)$ & $1.26(1.05,1.51)$ & - & \\
\hline$\geq 4.0$ & 676 & $238(35.2)$ & $1.35(1.10,1.66)$ & $\rightarrow$ & \\
\hline C-reactive protein, $\mathrm{mg} / \mathrm{L}$ & & & & & 0.042 \\
\hline$<11.1$ (quartile 3) & 712 & 239 (33.6) & $1.44(1.17,1.76)$ & $\rightarrow$ & \\
\hline$\geq 11.1$ & 239 & $110(46.0)$ & $1.04(0.80,1.35)$ & - & \\
\hline Albumin, $\mathrm{g} / \mathrm{L}$ & & & & & 0.714 \\
\hline$<40.0$ (median) & 728 & $302(41.5)$ & $1.27(1.05,1.53)$ & - & \\
\hline$\geq 40.0$ & 783 & $210(26.8)$ & $1.33(1.09,1.62)$ & $\rightarrow$ & \\
\hline Diabetes ${ }^{b}$ & & & & & 0.787 \\
\hline No & 982 & $285(29.0)$ & $1.31(1.08,1.59)$ & $\rightarrow$ & \\
\hline Yes & 652 & $268(41.1)$ & $1.26(1.03,1.55)$ & - & \\
\hline Baseline CVD & & & & & 0.482 \\
\hline No & 935 & 261 (27.9) & $1.22(0.98,1.53)$ & $=-$ & \\
\hline Yes & 699 & $292(41.8)$ & $1.35(1.13,1.60)$ & $\rightarrow$ & \\
\hline & & & & 11.52 & \\
\hline
\end{tabular}

Fig. 2. Relation of baseline SAF (per $1 \mathrm{AU}$ ) with the risk of all-cause mortality in various groups. ${ }^{a}$ Adjusted, if not stratified, for study center, age, sex, smoking status, BMI, SBP, dialysis vintage, albumin, hemoglobin, iPTH, phosphate, calcium, sodium, potassium, FG, TC, CVD status, and the usage of RAAS inhibitors. b Diabetes

sue, but not circulating, AGEs were related to cardiovascular surrogate, such as tissue velocity imaging on echocardiography [27] and low circulating endothelial progenitor cells [28]. Second, our results showed that dialysis vintage and CRP levels significantly modified the association between tissue AGEs and the risk of mortality among HD patients. A stronger association was found in those with shorter dialysis vintage or lower CRP levels. It has been recognized that the mortality-related confounders would increase as the increment of dialysis vintage and inflammatory condition. It is possible that the detrimental effect of tissue AGEs accumulation may be affected by some unidentified confounders. Our studies underscore the importance of measuring the SAF in the early stage of dialysis and suggest that the optimal control of was defined as an $\mathrm{FG} \geq 7.0 \mathrm{mmol} / \mathrm{L}$ or using of glucose-lowering drugs or having history of diabetes. SAF, skin autofluorescence; CVD, cardiovascular disease; SBP, systolic blood pressure; $\mathrm{PTH}$, intact parathyroid hormone; FG, fasting glucose; TC, total cholesterol; RAAS, renin-angiotensin-aldosterone system. tissue AGE accumulation may be a strategy for improving the outcomes in HD patients.

The potential mechanisms by which tissue accumulation of AGEs increases mortality risk are unclear, but it is biologically plausible. Accumulation of AGEs may lead to cross-links between structural proteins including collagen and elastin in arterial walls, resulting in arterial stiffness [29]. AGEs bind to its receptor (RAGE), alter signaling cascades, and provoke endothelial dysfunction [30], inflammatory responses, and oxidative stress [31, 32]. Consistently, higher SAF has been reported to be independently associated with arterial stiffness [33] and endothelial dysfunction determined by flow-mediated dilatation [34].
14

Am J Nephrol 2021;52:8-16

DOI: $10.1159 / 000512385$
Jiang et al. 
The current study has some limitations. First, although the regression models were adjusted for a broad array of covariates, residual confounding from unmeasured factors cannot be excluded. Second, our analyses were based on a single baseline measurement of SAF and CML and therefore may not reflect levels over a longer period. Third, since SAF measurements had only been validated in subjects with Fitzpatrick skin types 1-4, the results of the present study may not generalize to patients with other skin types. In addition, skin disorders, such as pallor, hyperpigmentation, and xerosis, are common and diverse in HD patients [35-37]. However, we did not collect the detailed information about those skin disorders in the current study. Therefore, although corrections using differences in skin reflectance may resolve confounding effects of skin pigmentation on SAF [38], we could not have a detailed analysis to evaluate the possible effect of the skin disorders on the SAF measurements. Fourth, we did not have available data on BUN, glycohemoglobin, and glycated albumin. Finally, due to the modestly improved C-statistics, NRI and IDI, the predictive performance and the clinical usefulness of measuring SAF should be further evaluated and confirmed in more studies. In summary, the tissue level of AGEs measured by SAF was associated with the risk of all-cause and CVD mortality among HD patients, especially in those with shorter dialysis vintage or lower CRP levels.

\section{Statement of Ethics}

Our study was approved by the local Ethics Committee in each center, and all participants provided written informed consent.

\section{Conflict of Interest Statement}

The authors declare that they have no competing interests.

\section{Funding Sources}

This study was funded by the National Natural Science Foundation of China (81470995 to J.P.J.), the Nature Science Foundation of Guangdong Province (2014A030313345 to J.P.J.), the Clinical Innovation Research Program of Guangzhou Regenerative Medicine and Health Guangdong Laboratory (2018GZR0201003 to F.F.H.), the Research Fund Program of Guangdong Provincial Key Laboratory of Renal Failure Research (2017B030314036 to F.F.H.), the Major International (Regional) Joint Research Project (81620108003 to F.F.H.), and the National Innovation Team Program (81521003 to Y.H.L).

\section{Author Contributions}

Study concept and design: F.F.H. and J.J. Conduct of study: F.F.H., J.J., J.C., X.Y., C.M., F.X., W.S., W.Z., X.L., S.S., P.Z., Y.Z., Y.Z., S.L., Z.Z., Q.L., Y.Y., J.T., and W.L. Data collection and analysis: J.J., Y.Z., and X.Q. Drafting of the manuscript: J.J., Y.Z., and X.Q. Critical review and revision of the manuscript: F.F.H.

\section{References}

1 Goodkin DA, Young EW, Kurokawa K, Prütz KG, Levin NW. Mortality among hemodialysis patients in Europe, Japan, and the United States: case-mix effects. Am J Kidney Dis. 2004;44(5 Suppl 2):16-21.

2 Go AS, Chertow GM, Fan D, McCulloch CE, Hsu CY. Chronic kidney disease and the risks of death, cardiovascular events, and hospitalization. N Engl J Med. 2004;351(13):1296305.

3 Pozzoni P, Del Vecchio L, Pontoriero G, Di Filippo S, Locatelli F. Long-term outcome in hemodialysis: morbidity and mortality. J Nephrol. 2004;17(Suppl 8):S87-95.

4 Melamed ML, Eustace JA, Plantinga L, Jaar BG, Fink NE, Coresh J, et al. Changes in serum calcium, phosphate, and PTH and the risk of death in incident dialysis patients: a longitudinal study. Kidney Int. 2006;70(2): $351-7$.

5 Nakashima A, Carrero JJ, Qureshi AR, Miyamoto T, Anderstam B, Bárány P, et al. Effect of circulating soluble receptor for advanced glycation end products (sRAGE) and the proinflammatory RAGE ligand (EN-RAGE,
S100A12) on mortality in hemodialysis patients. Clin J Am Soc Nephrol. 2010;5(12): 2213-9.

6 Thorpe SR, Baynes JW. Maillard reaction products in tissue proteins: new products and new perspectives. Amino Acids. 2003;25(34):275-81.

7 Agalou S, Ahmed N, Babaei-Jadidi R, Dawnay A, Thornalley PJ. Profound mishandling of protein glycation degradation products in uremia and dialysis. J Am Soc Nephrol. 2005; 16(5):1471-85.

8 Meerwaldt R, Zeebregts CJ, Navis G, Hillebrands JL, Lefrandt JD, Smit AJ. Accumulation of advanced glycation end products and chronic complications in ESRD treated by dialysis. Am J Kidney Dis. 2009;53(1):138-50.

9 Schwedler SB, Metzger T, Schinzel R, Wanner C. Advanced glycation end products and mortality in hemodialysis patients. Kidney Int. 2002;62(1):301-10.

10 Suliman ME, Heimbürger O, Bárány P, Anderstam B, Pecoits-Filho R, Rodríguez Ayala $\mathrm{E}$, et al. Plasma pentosidine is associated with inflammation and malnutrition in end-stage renal disease patients starting on dialysis therapy. J Am Soc Nephrol. 2003;14(6):161422.

11 Wagner Z, Molnár M, Molnár GA, Tamaskó M, Laczy B, Wagner L, et al. Serum carboxymethyllysine predicts mortality in hemodialysis patients. Am J Kidney Dis. 2006;47(2): 294-300.

12 Arsov S, Graaff R, van Oeveren W, Stegmayr B, Sikole A, Rakhorst G, et al. Advanced glycation end-products and skin autofluorescence in end-stage renal disease: a review. Clin Chem Lab Med. 2014;52(1):11-20.

13 Meerwaldt R, Graaff R, Oomen PHN, Links TP, Jager JJ, Alderson NL, et al. Simple noninvasive assessment of advanced glycation endproduct accumulation. Diabetologia. 2004;47(7):1324-30.

14 Jiang J, Chen P, Chen J, Yu X, Xie D, Mei C, et al. Accumulation of tissue advanced glycation end products correlated with glucose exposure dose and associated with cardiovascular morbidity in patients on peritoneal dialysis. Atherosclerosis. 2012;224(1):18794. 
15 Hou F, Jiang J, Chen J, Yu X, Zhou Q, Chen $\mathrm{P}$, et al. China collaborative study on dialysis: a multi-centers cohort study on cardiovascular diseases in patients on maintenance dialysis. BMC Nephrol. 2012;13:94.

16 Zhou QG, Jiang JP, Wu SJ, Tian JW, Chen JH, Yu XQ, et al. Current pattern of Chinese dialysis units: a cohort study in a representative sample of units. Chin Med J. 2012;125(19): 3434-9.

17 Zhou Q, Wu S, Jiang J, Tian J, Chen J, Yu X, et al. Accumulation of circulating advanced oxidation protein products is an independent risk factor for ischaemic heart disease in maintenance haemodialysis patients. Nephrology. 2012;17(7):642-9.

18 Henle T. Protein-bound advanced glycation endproducts (AGEs) as bioactive amino acid derivatives in foods. Amino Acids. 2005; 29(4):313-22.

19 Arsov S, Trajceska L, van Oeveren W, Smit AJ, Dzekova P, Stegmayr B, et al. Increase in skin autofluorescence and release of hearttype fatty acid binding protein in plasma predicts mortality of hemodialysis patients. Artif Organs. 2013;37(7):E114-22.

20 Meerwaldt R, Hartog JW, Graaff R, Huisman RJ, Links TP, den Hollander NC, et al. Skin autofluorescence, a measure of cumulative metabolic stress and advanced glycation end products, predicts mortality in hemodialysis patients. J Am Soc Nephrol. 2005;16(12): 3687-93.

21 Nongnuch A, Davenport A. Skin autofluorescence advanced glycosylation end products as an independent predictor of mortality in high flux haemodialysis and haemodialysis patients. Nephrology. 2015;20(11):862-7.

22 Gerrits EG, Lutgers HL, Smeets GH, Groenier $\mathrm{KH}$, Smit AJ, Gans RO, et al. Skin autofluorescence: a pronounced marker of mortality in hemodialysis patients. Nephron Extra. 2012; 2(1):184-91.
23 Viramontes Hörner D, Selby NM, Taal MW. Skin autofluorescence and malnutrition as predictors of mortality in persons receiving dialysis: a prospective cohort study. J Hum Nutr Diet. 2020 Dec;33(6):852-61.

24 Kimura H, Tanaka K, Kanno M, Watanabe K, Hayashi Y, Asahi K, et al. Skin autofluorescence predicts cardiovascular mortality in patients on chronic hemodialysis. Ther Apher Dial. 2014;18(5):461-7.

25 Mukai H, Svedberg O, Lindholm B, Dai L, Heimbürger O, Barany $\mathrm{P}$, et al. Skin autofluorescence, arterial stiffness and Framingham risk score as predictors of clinical outcome in chronic kidney disease patients: a cohort study. Nephrol Dial Transplant. 2019;34(3): 442-8.

26 Cavero-Redondo I, Soriano-Cano A, ÁlvarezBueno C, Cunha PG, Martínez-Hortelano JA, Garrido-Miguel M, et al. Skin autofluorescence -indicated advanced glycation end products as predictors of cardiovascular and all-cause mortality in high-risk subjects: a systematic review and meta-analysis. J Am Heart Assoc. 2018;7(18):e009833.

27 Hartog JW, Hummel YM, Voors AA, Schalkwijk CG, Miyata T, Huisman RM, et al. Skinautofluorescence, a measure of tissue advanced glycation end-products (AGEs), is related to diastolic function in dialysis patients. J Card Fail. 2008;14(7):596-602.

28 Ueno H, Koyama H, Fukumoto S, Tanaka S, Shoji T, Shoji T, et al. Advanced glycation end products, carotid atherosclerosis, and circulating endothelial progenitor cells in patients with end-stage renal disease. Metab Clin Exp. 2011;60(4):453-9.

29 Aronson D. Cross-linking of glycated collagen in the pathogenesis of arterial and myocardial stiffening of aging and diabetes. J Hypertens. 2003;21(1):3-12.
30 Linden E, Cai W, He JC, Xue C, Li Z, Winston $\mathrm{J}$, et al. Endothelial dysfunction in patients with chronic kidney disease results from advanced glycation end products (AGE)-mediated inhibition of endothelial nitric oxide synthase through RAGE activation. Clin J Am Soc Nephrol. 2008;3(3):691-8.

31 Maillard-Lefebvre $\mathrm{H}$, Boulanger E, Daroux M, Gaxatte C, Hudson BI, Lambert M. Soluble receptor for advanced glycation end products: a new biomarker in diagnosis and prognosis of chronic inflammatory diseases. Rheumatology. 2009;48(10):1190-6.

32 Prasad K, Mishra M. AGE-RAGE stress, stressors, and antistressors in health and disease. Int J Angiol. 2018;27(1):1-12.

33 Ueno H, Koyama H, Tanaka S, Fukumoto S, Shinohara K, Shoji T, et al. Skin autofluorescence, a marker for advanced glycation end product accumulation, is associated with arterial stiffness in patients with end-stage renal disease. Metab Clin Exp. 2008;57(10):1452-7.

34 Wang C-C, Wang Y-C, Wang G-J, Shen M-Y, Chang Y-L, Liou S-Y, et al. Skin autofluorescence is associated with endothelial dysfunction in uremic subjects on hemodialysis. PLoS One. 2016;11(1):e0147771.

35 Khanna D, Singal A, Kalra OP. Comparison of cutaneous manifestations in chronic kidney disease with or without dialysis. Postgrad Med J. 2010;86(1021):641-7.

36 Solak B, Acikgoz SB, Sipahi S, Erdem T. Cutaneuos findings in patients with predialysis chronic kidney disease. J Eur Acad Dermatol Venereol. 2016;30(9):1609-13.

37 Kuypers DR. Skin problems in chronic kidney disease. Nat Clin Pract Nephrol. 2009;5(3): 157-70.

38 Meerwaldt R, Links T, Graaff R, Thorpe SR, Baynes JW, Hartog J, et al. Simple noninvasive measurement of skin autofluorescence. Ann N Y Acad Sci. 2005;1043:290-8. 